\title{
(- OPEN ACCESS \\ New advances in amblyopia therapy I: binocular therapies and pharmacologic augmentation
}

\author{
Courtney L Kraus, ${ }^{1}$ Susan M Culican ${ }^{2}$
}

'Wilmer Eye Institute, Johns Hopkins University, Baltimore, Maryland, USA ${ }^{2}$ Department of Ophthalmology and Visual Sciences, Washington University School of Medicine, St. Louis, Missouri, USA

\section{Correspondence to} Dr Courtney L Kraus, Wilmer Eye Institute, Johns Hopkins University, Baltimore, MD 21287, USA; ckraus6@jhmi.edu

Received 28 February 2018 Revised 16 April 2018 Accepted 22 April 2018 Published Online First 18 May 2018

\section{ABSTRACT}

Amblyopia therapy options have traditionally been limited to penalisation of the non-amblyopic eye with either patching or pharmaceutical penalisation. Solid evidence, mostly from the Pediatric Eye Disease Investigator Group, has validated both number of hours a day of patching and days per week of atropine use. The use of glasses alone has also been established as a good first-line therapy for both anisometropic and strabismic amblyopia. Unfortunately, visual acuity equalisation or even improvement is not always attainable with these methods. Additionally, non-compliance with prescribed therapies contributes to treatment failures, with data supporting difficulty adhering to full treatment sessions. Interest in alternative therapies for amblyopia treatment has long been a topic of interest among researchers and clinicians alike. Incorporating new technology with an understanding of the biological basis of amblyopia has led to enthusiasm for binocular treatment of amblyopia. Early work on perceptual learning as well as more recent enthusiasm for iPadbased dichoptic training have each generated interesting and promising data for vision improvement in amblyopes. Use of pharmaceutical augmentation of traditional therapies has also been investigated. Several different drugs with unique mechanisms of action are thought to be able to neurosensitise the brain and enhance responsiveness to amblyopia therapy. No new treatment has emerged from currently available evidence as superior to the traditional therapies in common practice today. But ongoing investigation into the use of both new technology and the understanding of the neural basis of amblyopia promises alternate or perhaps better cures in the future.

\section{INTRODUCTION}

Amblyopia therapy has benefited significantly from the investigations of the Paediatric Eye Disease Investigator Group (PEDIG). There have been 18 completed Amblyopia Treatment Studies (ATS) as of publication of this review. In these well-designed, predominantly randomised controlled trials, there have been numerous notable conclusions that continue to shape and dictate how to care for children with amblyopia. Early studies provided solid epidemiological data on patients with amblyopia. ${ }^{1-3}$ Through the early studies, the equivalent efficacy of patching versus atropine penalisation was established. ${ }^{4-6}$ Concern that cessation of treatment would lead to a relapse in amblyopia was widely touted, but 15 -year follow-up data confirmed that the amblyopia treatment effect persisted. ${ }^{7}$
Traditional amblyopia treatment strategies have documented improvement with spectacle correction when indicated, followed by patching or atropine penalisation of the non-amblyopic ('fellow') eye. While the majority of children show improvement with these approaches, not all children respond to traditional therapies. Even responders often have residual amblyopia. Fifty-four per cent of children treated at age $<3-7$ years still demonstrate some amblyopia at age $10 .^{4}$ Older children fair even worse; 74\% of children aged 7-12 years treated with patching, and $80 \%$ treated with atropine have some degree of residual amblyopia on long-term follow-up. ${ }^{5}$ In the teenage cohort, outcomes are even less effective with only one-quarter to one-half of children responding to combined treatment of spectacles and patching depending on whether they had previous treatment or were treatment naïve (respectively). ${ }^{6}$

Non-compliance contributes to treatment failures, with data supporting less than perfect adherence to prescribed regimens. ${ }^{8}$ However, data from use of an occlusion dose monitor confirmed that some children demonstrate excellent compliance, yet still fail to improve. ${ }^{910}$ This suggests an opportunity for novel strategies to target non-compliant patients and non-responders. In this review, we will explore the binocular therapies as an alternative to traditional amblyopia treatments, as well as pharmacologic adjuncts to standard regimens.

\section{BINOCULAR THERAPIES}

\section{Background and rationale}

The depth of amblyopia has been positively correlated to the degree of binocular imbalance. ${ }^{11}$ Affected individuals show impaired stereoacuity and abnormal binocular summation. ${ }^{12}$ Yet evidence suggests that binocular cortical communication persists in subjects with amblyopia. ${ }^{13} 14$ These findings are the basis for the hypothesis that activation of these persistent binocular neural circuits might be exploited to 'awaken' an amblyopic eye. Binocular therapies designed to improve amblyopia through binocular stimulation are largely broken down into perceptual learning and dichoptic training.

\section{Perceptual learning}

Perceptual learning was defined in 1963 by Eleanor Gibson as an evolution in the discernment of a stimulus array after repetitive exposure or practice with this array. ${ }^{15}$ This work is the psychophysical validation of the old adage 'Practice makes perfect'. Performance on simple visual tasks has been long known to improve with practice in adults. Application 
of perceptual learning to various visual tasks has reportedly resulted in improvement in several measures, including orientation discrimination, stereoacuity and contrast sensitivity. ${ }^{16} \mathrm{~A}$ number of visual tasks have been explored as a means to apply perceptual learning, including vernier acuity, Gabor detection, positional discrimination, letter identification in noise, position discrimination in noise and contrast detection. ${ }^{17}$

Studies by Polat et al suggest that perceptual learning in adult amblyopes can augment visual function. Improved pretest to post-test performance and gains in visual acuity (VA) were reported when subjects participated in a learnt trial of Gabor signals in a series of 77 adult amblyopes. ${ }^{18}$ The criticism of this approach is that gains on test outcome measures in the amblyopic eye do not transfer to novel situations-improvement is only seen for the task practised. ${ }^{19}$ Advocates of perceptual learning note that the specific nature of the stimuli chosen for training tasks contributes to the capacity for generalisability of the trained discrimination. ${ }^{20}$ Others cite the targeted reduction in the detrimental effect of crowding (a reduction in VA when viewing a line of linear letters more severe than when viewing letters individually). ${ }^{21}$ The neural basis for this is postulated to result from a reduction in lateral inhibition within the brain with training. $^{22}$

Small studies of juvenile amblyopes have demonstrated improvements. Seven participants with prior occlusion therapy had improved visual performance following completion of a positional discrimination task. ${ }^{23}$ A second pilot study of five amblyopic children who underwent 40 hours of perceptual learning demonstrated improved scores on Snellen acuity and contrast sensitivity. There was no follow-up following completion of the treatment regimen. ${ }^{24}$ In their comprehensive review of perceptual learning, Levi and Li reported on the relative effectiveness of the various types of tasks in both performance of the trained task and Snellen acuity. Five of the 12 studies reviewed showed improvement in post-test results, of which 4 employed practising contrast detection. The fifth study examined extended positional acuity learning in children. ${ }^{17} 25$
Drawbacks to treatment

Perceptual learning has yet to gain widespread support. Most of the aforementioned studies contained very small numbers of participants, limiting generalisability to populations at large. Perceptual learning effects have been demonstrated to last hours to months without continued practice, ${ }^{26}$ but long-term follow-up is lacking. Additionally, implementation of a successful clinical programme of treatment would require the ability to perform training at home while the aforementioned studies required perceptual learning tasks to be in a laboratory setting.

\section{Dichoptic training}

Unlike perceptual learning, where a single visual percept is administered to both eyes simultaneously or under monocular viewing conditions, dichoptic treatment presents independent stimuli to each eye (figure 1). The therapy derives its effect from unlocking binocular visual function. The treatment effect then follows from introducing a task that requires the integration of the two stimuli under binocular viewing conditions. The paradigm is customised to overcome the patient's suppression of the amblyopic eye. To do so, the image shown to the amblyopic eye must be of higher contrast than that shown to the fellow eye. ${ }^{14}$

${ }^{27}$ As the patient's developing binocular function improves, the contrast difference between the two eyes is reduced, potentially to a point where no difference is required. VA gains follow improvements in binocularity and contrast sensitivity, presumably due to reduced suppression. ${ }^{24}$

Early reports of dichoptic training used this concept in a clinic-based setting with the adult amblyopes. ${ }^{28-30}$ They demonstrated both proof-of-concept as well as the potential for amblyopia improvement outside of the critical period. Hess et al showed statistically significant improvements in amblyopic eye visual and stereoacuity in nine adults (four of whom had prior patching treatment). ${ }^{28}$

In order to move the therapy from a clinic-based, observed task to mobile, home-based use, it was necessary to adapt the design to be more user-friendly. An iPod display with a lenticular

\section{DE (LE)}

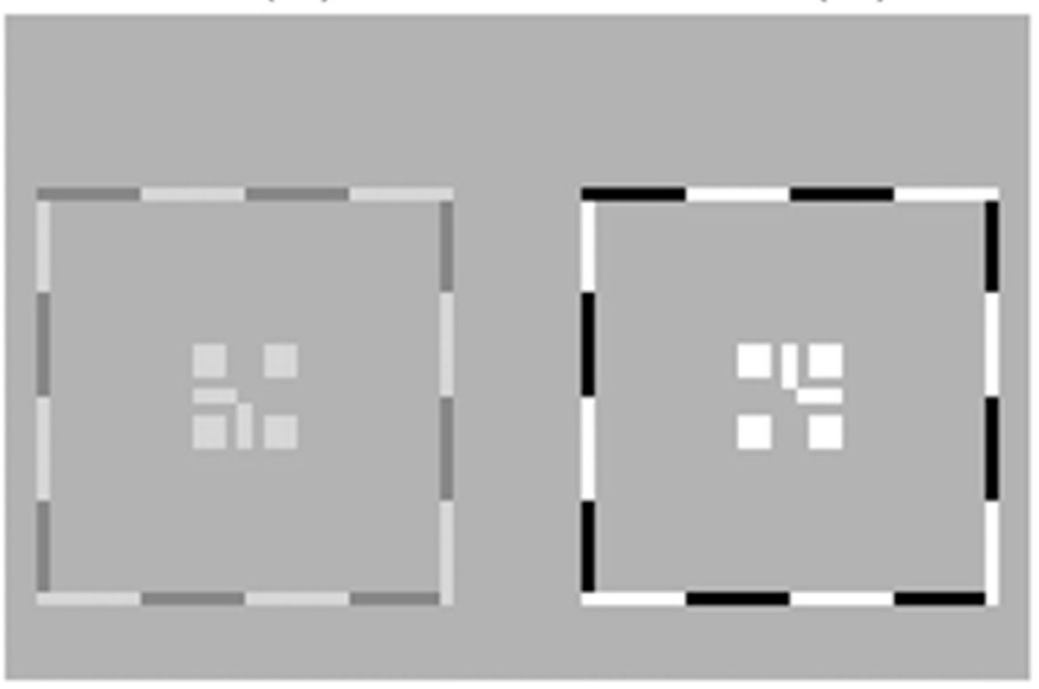

$\mathrm{DE}+\mathrm{NDE}(\mathrm{LE}+\mathrm{RE})$

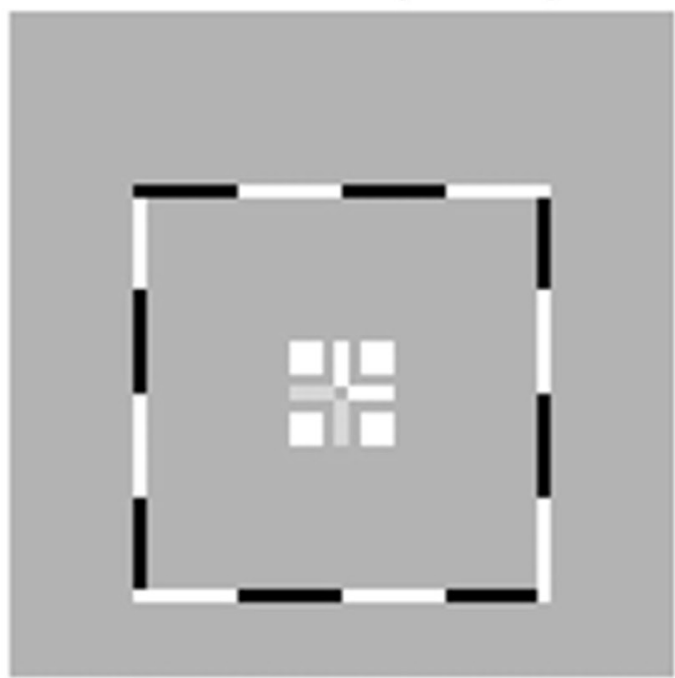

Figure 1 Dichoptic stimuli as presented to the patient with amblyopia. The stimuli are adjusted so that the dominant eye (DE), in this case the left eye (LE), has less contrast and is therefore more difficult to discriminate than the non-dominant eye (NDE). When the images are superimposed, the subject perceives a single percept with summation of elements presented to each eye separately. Over time, the contrast can be adjusted as the nondominant eye improves with training (reproduced from Ding and Levi, figure $1 \mathrm{~A}$ ) ${ }^{62} \mathrm{RE}$, right eye. 
overlay (a textured screen overlay that produces images with a perception of depth, eg, holograms) allowed for viewing the dichoptic stimuli, but required stable head positioning. While studies suggest that such method is successful, it is a challenge to use. ${ }^{31}$ A shift away from the lenticular design and transition to anaglyphic (red-green dichoptic) images with accompanying red-green glasses overcame this limitation and permitted utilisation of this form of therapy by young children and in unsupervised patients. ${ }^{29} 32$

\section{Binocular iPad therapy}

Interest in dichoptic treatment progressed with the migration of the Hess Falling Blocks game onto an iPad. Birch and colleagues conducted a small study of children aged 4-12 playing this dichoptic iPad game using red-green anaglyphic glasses for 4 hours/week over 4 weeks and saw improvement in amblyopic eye $\log$ MAR acuity $(0.47-0.39, \mathrm{p}<0.001) .{ }^{33}$ A subsequent study looking at younger children confirmed previous work demonstrating improved amblyopic-eye acuity as well as a dose-response effect. ${ }^{32}$ Those children completing 8 total hours of game play during the 4-week study had significantly greater improvement than those playing $0-4$ hours. The results of these early studies suggested promise for dichoptic training in the treatment of amblyopia.

In 2015, PEDIG conducted the first large-scale, multisite randomised controlled trial comparing the effectiveness of 1 hour/day, 7 days/week binocular game play to 2 hours/day patching in children $<13$ as a non-inferiority study. There was a parallel superiority study examining the same regimen in children aged 13 to $<17$ years. Results of the non-inferiority study in the younger cohort demonstrated improvement in both the 1 hour/day iPad game play as well as the 2 hours/day patching groups with no statistically significant difference between the groups at 16 weeks. There were no side effects of treatment, specifically diplopia, reduction in fellow eye VA or new tropia. A disappointing finding of the study was the poor overall compliance in the binocular game group. ${ }^{34}$ The 13 to $<17$ years age group cohort results were similar; amblyopic eye VA was not better with iPad play, and was possibly worse. Compliance was similarly poor, with $13 \%$ completing $>75 \%$ of prescribed treatment. ${ }^{35}$

Similar work by Gao et al compared 1 hour of home-based, dichoptic falling-blocks video game play to a placebo game. ${ }^{36}$ They recruited participants age 7 years and up, including adults 18 years and older. Results failed to show a meaningful difference in amblyopic eye 6-week VA, the primary outcome of interest. They found no significant age effect, type of amblyopia or impact of prior occlusion treatment.

\section{Drawbacks to treatment}

While initially heralded as superior to patching due to theorised improved compliance, the results of the first randomised controlled trial were underwhelming. Less than one-quarter of children in the PEDIG study completed $75 \%$ of prescribed treatment time. ${ }^{34}$ Thus, ironically, non-compliance has dampened enthusiasm for binocular amblyopia treatment over traditional therapies. The theorised rationale for the high rate of non-compliance is that the Falling Blocks game was not stimulating enough to encourage a full hour of play on multiple days per week. The author's own experience included patient reports of preference for patching due to the wider range of activities that could be performed with a patch over the repetitive play on the iPad game. Similarly, poor compliance and participant dropout was seen in weeks 4-6 in the study by Gao et al.$^{36}$ This led the authors to suggest the need for more engaging games with potential reward reinforcement as the next iteration of binocular iPad play. Use of a more stimulating Dig Rush game has shown promise ${ }^{37}$ and recruitment is currently underway for a randomised controlled trial comparing play with glasses with glasses alone (NCT02983552).

\section{Alternative technologies}

While iPad-based platforms for binocular treatment of amblyopia have the most research into their use incorporating a variety of study designs and age groups, alternative technological presentations of binocular therapy have been created. Passive viewing of dichoptic movies has demonstrated success in a paediatric cohort both with compliance and vision outcomes. Study limitations included short, 2-week follow-up and lack of randomised design. ${ }^{38}$ Head-mounted virtual reality displays have also shown preliminary evidence to suggest improvement in both VA and stereoacuity in adult patients ${ }^{39}$ as has video game play. ${ }^{40}{ }^{41}$ This latter study by Vedamurthy et al also demonstrated retention of VA and stereoacuity after a 2 -month time period. ${ }^{41}$ Continuously evolving technology will likely yield additional means to deliver dichoptic stimuli in engaging, interactive platforms; hopefully, with the added benefit of effectiveness and patient compliance.

Interactive binocular treatment (I-BiT) system was developed to treat amblyopia using dichoptic stimuli presented via virtual reality game play or movie watching. ${ }^{42}$ The special software selectively stimulates the amblyopic eye without compromising vision in the fellow eye. Initial pilot studies showed promise improving the VA of paediatric and adult patients with amblyopia. ${ }^{4-45}$ Use of shutter glasses has also been paired with this technology, where the glasses lighten and darken in synchrony with the monitor, allowing an enriched image to be presented to the amblyopic eye only. ${ }^{4166}$ In one study, all subjects used shutter glasses to present dichoptic stimuli but were randomised to one of three arms: active I-BiT game play, passive I-BiT DVD use or non-I-BiT game play. There was improvement in all groups in VA, with no meaningful difference found between the groups. Interestingly, the game platform that included an interactive shooter game and DVD, had $>90 \%$ participant-reported satisfaction with treatments. ${ }^{46}$ This lends further support to the notion of patient engagement in treatment success or compliance.

\section{THE DRUG THERAPIES \\ Background and rationale}

Amblyopia is considered to be most receptive to treatments initiated within the 'critical period', during which cortical brain plasticity allows for reversal of some or all of the visual loss in the non-dominant eye. Results of the ATS have shown that the critical period is protracted with visual improvement possible up to age 17 years. However, the response to treatment is greatest under age 7 years, with waning benefit with increasing age. Furthermore, treatment during the teen years disproportionately benefits those with no prior history of treatment. ${ }^{47}$ The opportunity to neurosensitise a brain to allow for improvement with patching or atropine in children for whom conventional treatments have failed or after the critical period has ended is desirable. Pharmaceutical agents may offer that ability and a select few have reached human studies.

\section{Pharmacologic options: levodopa-carbidopa}

A theory has been proposed that increasing levels of dopamine may improve vision in the context of amblyopia. Some 
investigators have reported that levels of retinal dopamine are decreased in deprivation amblyopia. ${ }^{48}$ The first report of dopamine augmentation came in 1990 when Gottlob and Stangler-Zuschrott examined the effect of levodopa on adult amblyopia. ${ }^{49}$ Levodopa is the immediate metabolic precursor of dopamine and is Food and Drug Administration approved for use in other neurological disorders.

There have been several clinical trials that have evaluated the use of levodopa across a range of patients. PEDIG investigators organised a randomised trial of levodopa for the treatment of amblyopia in an older cohort of patients (children aged 7-12 years). When prescribed daily levodopa with carbidopa in addition to continued 2 hours/day of patching, no clinically significant or meaningful improvement in VA was seen. Reassuringly, no serious adverse events were reported. ${ }^{50}$ In a different prospective trial with a larger cohort of patients, children who had previously received spectacles but were otherwise treatment-naïve were prescribed full-time patching and then randomised to levodopa or placebo. The authors reported statistically significant visual gains sustained at 1 year of follow-up for children in the levodopa group. In this study, the levodopa dosage was three times higher than in the PEDIG study. ${ }^{51}$

\section{Pharmacologic options: citicoline}

Citicoline is a complex biomolecule involved in cellular metabolism. Its structure confers both cholinergic and neuroprotective properties. ${ }^{52}$ Due to its role in phospholipid metabolism, citicoline has been theorised to protect the anatomic and structural integrity of cell membranes, thereby preventing nerve cell damage. This has led to its use for the recovery from traumatic, ischaemic and degenerative insults. ${ }^{53}$ It was initially trialled in ophthalmic care for the treatment of glaucoma. ${ }^{54}$

Initial work in adult patients demonstrated improvement in VA with citicoline augmentation of patching that was not sustained following cessation of the medication. ${ }^{55}$ Early studies in amblyopic children were promising, showing treatment effect with citicoline both alone and in addition to patching. ${ }^{56} 57$ A study of treatment-naïve participants randomised to added citicoline after a run-in patching phase showed a significant treatment effect at 90 days for the citicoline-augmented group. ${ }^{58}$ However, failure to demonstrate improvement in the control group ( 2 hours a day of patching) was unexpected and therefore results from this study should be cautiously interpreted.

Research into the use of citicoline is arguably behind that of levodopa. Well-designed randomised controlled trials and appropriately selected treatment groups need to be initiated. At the time of this review, all the studies of citicoline failed to include follow-up periods beyond 3-6 months.

\section{Drawbacks to medical therapy}

Pharmaceutical augmentation of amblyopia therapy appears to be well tolerated. A liquid suspension of levodopa is available to facilitate use in a young patient population, although has a reportedly unpleasant bitter taste. ${ }^{59} 60$ Side effects of levodopa therapy are reassuringly mild, with children describing mild nausea, vomiting and headache ${ }^{3132}$ and not severe enough to necessitate cessation of treatment. The addition of carbidopa to the prescribed formulation reduces these gastrointestinal side effects by inhibiting peripheral conversion of levodopa to dopamine. Because carbidopa cannot cross the blood-brain barrier, it only prevents levodopa conversion peripherally and allows more central activity of levodopa. One worrisome result from the PEDIG study was regression of treatment effect with drug cessation. ${ }^{31}$ Therefore, randomised controlled trials with ample follow-up still remain necessary. Side effects of citicoline were negligible in all studies. In early use, intramuscular injection was the only means of administration; however, there is now an oral formation. ${ }^{61}$ Medical therapy, in isolation or in addition to conventional therapy, is still in its infancy and potential agents are in the research and development stages.

\section{SUMMARY/CONCLUSIONS}

The past 15 years have been replete with well-designed, prospective controlled clinical trials to demonstrate both the efficacy and limitations of traditional amblyopia therapies. Novel approaches to this problem have met with mixed success. Perceptual learning and medical intervention have shown promise, but lack well-designed studies to suggest sustained effect outside the treatment period. Dichoptic training has extensive research suggesting effectiveness, but the most recent randomised trial failed to demonstrate non-inferiority over standard treatments. Future investigation will likely continue to modify and adapt these novel approaches to generative creative, engaging amblyopia therapies that may benefit children and adults, alike.

Contributors CLK wrote the first draft of the review. SMC edited and added relevant references and citations. Both authors approved the final version of the manuscript.

Funding This work was supported by awards to the Department of Ophthalmology and Visual Sciences at Washington University from a Research to Prevent Blindness, unrestricted grant (New York, New York), the NIH Vision Core Grant P30 EY 0268 (Bethesda, Maryland). The funding organisations had no role in the design or conduct of this research.

Competing interests None declared.

Patient consent Not required.

Provenance and peer review Not commissioned; externally peer reviewed.

Open access This is an Open Access article distributed in accordance with the Creative Commons Attribution Non Commercial (CC BY-NC 4.0) license, which permits others to distribute, remix, adapt, build upon this work non-commercially, and license their derivative works on different terms, provided the original work is properly cited and the use is non-commercial. See: http://creativecommons.org/ licenses/by-nc/4.0/

(c) Article author(s) (or their employer(s) unless otherwise stated in the text of the article) 2018. All rights reserved. No commercial use is permitted unless otherwise expressly granted.

\section{REFERENCES}

1 Pediatric Eye Disease Investigator Group. The clinical profile of moderate amblyopia in children younger than 7 years. Arch Ophthalmol 2002;120:281-7.

2 Pediatric Eye Disease Investigator Group. The course of moderate amblyopia treated with patching in children: experience of the amblyopia treatment study. Am J Opthalmol 2003:136:620-9.

3 Pediatric Eye Disease Investigator Group. The course of moderate amblyopia treated with atropine in children: experience of the amblyopia treatment study. Am J Opthalmol 2003;136:630-9.

4 Repka MX, Kraker RT, Beck RW, et al. Pediatric Eye Disease Investigator Group. A randomized trial of atropine vs patching for treatment of moderate amblyopia: followup at age 10 years. Arch Ophthalmol 2008;126:1039-44.

5 Scheiman MM, Hertle RW, Kraker RT, et al. Pediatric Eye Disease Investigator Group. Patching vs atropine to treat amblyopia in children aged 7 to 12 years: a randomized trial. Arch Ophthalmol 2008;126:1634-42.

6 Scheiman MM, Hertle RW, Beck RW, et al. . Randomized trial of treatment of amblyopia in children aged 7 to 17 years. Arch Ophthalmol 2005;123:437-47.

7 Pediatric Eye Disease Investigator Group. A Randomized Trial of Atropine versus Patching for Treatment of Moderate Amblyopia: Follow-up at 15 Years of Age. JAMA Ophthalmology 2014;132:799-805.

8 Oliver M, Neumann R, Chaimovitch Y, et al. Compliance and results of treatment for amblyopia in children more than 8 years old. Am J Ophthalmol 1986;102:340-5.

9 Loudon SE, Polling JR, Simonsz HJ. Electronically measured compliance with occlusion therapy for amblyopia is related to visual acuity increase. Graefes Arch Clin Exp Ophthalmol 2003:241:176-80. 
10 Stewart CE, Moseley MJ, Stephens DA, et al. Treatment dose-response in amblyopia therapy: the Monitored Occlusion Treatment of Amblyopia Study (MOTAS). Invest Ophthalmol Vis Sci 2004;45:3048-54.

11 Hess RF, Thompson B. Amblyopia and the binocular approach to its therapy. Vision Res 2015;114:4-16.

12 Thompson B, Richard A, Churan J, et al. Impaired spatial and binocular summation for motion direction discrimination in strabismic amblyopia. Vision Res 2011;51:577-84.

13 Joly 0, Frankó E. Neuroimaging of amblyopia and binocular vision: a review. Front Integr Neurosci 2014;8:62.

14 Hess RF, Thompson B, Baker DH. Binocular vision in amblyopia: structure, suppression and plasticity. Ophthalmic Physiol Opt 2014;34:146-62.

15 Gibson EJ. Perceptual learning. Annu Rev Psychol 1963;14:29-56.

16 Crist RE, Li W, Gilbert CD. Learning to see: experience and attention in primary visual cortex. Nat Neurosci 2001;4:519-25.

17 Levi DM, Li RW. Perceptual learning as a potential treatment for amblyopia: a minireview. Vision Res 2009;49:2535-49.

18 Polat U, Ma-Naim T, Belkin M, et al. Improving vision in adult amblyopia by perceptual learning. Proc Natl Acad Sci U S A 2004;101:6692-7.

19 Levi DM, Polat U. Neural plasticity in adults with amblyopia. Proc Natl Acad Sci U SA 1996;93:6830-4.

20 Jeter PE, Dosher BA, Petrov A, et al. Task precision at transfer determines specificity of perceptual learning. J Vis 2009;9:1.

21 Hussain Z, Webb BS, Astle AT, et al. Perceptual learning reduces crowding in amblyopia and in the normal periphery. J Neurosci 2012;32:474-80.

22 Maniglia M, Pavan A, Cuturi LF, et al. Reducing crowding by weakening inhibitory lateral interactions in the periphery with perceptual learning. PLoS One 2011;6:e25568.

23 Li RW, Young KG, Hoenig P, et al. Perceptual learning improves visual performance in juvenile amblyopia. Invest Ophthalmol Vis Sci 2005;46:3161-8.

24 Polat U, Ma-Naim T, Spierer A. Treatment of children with amblyopia by perceptual learning. Vision Res 2009;49:2599-603.

25 Li RW, Provost A, Levi DM. Extended perceptual learning results in substantial recovery of positional acuity and visual acuity in juvenile amblyopia. Invest Ophthalmol Vis Sci 2007;48:5046-51.

26 Qu Z, Song Y, Ding Y. ERP evidence for distinct mechanisms of fast and slow visual perceptual learning. Neuropsychologia 2010;48:1869-74.

27 Mansouri B, Thompson B, Hess RF. Measurement of suprathreshold binocular interactions in amblyopia. Vision Res 2008;48:2775-84.

28 Hess RF, Babu RJ, Clavagnier S, et al. The iPod binocular home-based treatment for amblyopia in adults: efficacy and compliance. Clin Exp Optom 2014;97:389-98.

29 Hess RF, Mansouri B, Thompson B. A new binocular approach to the treatment of amblyopia in adults well beyond the critical period of visual development. Restor Neurol Neurosci 2010;28:793-802.

30 Li J, Thompson B, Deng D, et al. Dichoptic training enables the adult amblyopic brain to learn. Curr Biol 2013;23:R308-R309.

31 To L, Thompson B, Blum JR, et al. A game platform for treatment of amblyopia. IEEE Trans Neural Syst Rehabil Eng 2011;19:280-9.

32 Birch EE, Li SL, Jost RM, et al. Binocular iPad treatment for amblyopia in preschool children. J Aapos 2015;19:6-11.

33 Li SL, Jost RM, Morale SE, et al. A binocular iPad treatment for amblyopic children. Eye 2014;28:1246-53.

34 Holmes JM, Manh VM, Lazar EL, et al. . Effect of a Binocular iPad Game vs Part-time Patching in Children Aged 5 to 12 Years With Amblyopia: A Randomized Clinical Trial. JAMA Ophthalmol 2016;134:1391-400.

35 Manh VM, Holmes JM, Lazar EL, et al. Pediatric Eye Disease Investigator Group. A Randomized Trial of a Binocular iPad Game Versus Part-Time Patching in Children Aged 13 to 16 Years With Amblyopia. Am J Ophthalmol 2018;186:104-15.

36 Gao TY, Guo CX, Babu RJ, et al. . Effectiveness of a Binocular Video Game vs Placebo Video Game for Improving Visual Functions in Older Children, Teenagers, and Adults With Amblyopia: A Randomized Clinical Trial. JAMA Ophthalmol 2018;136:172-81.

37 Kelly KR, Jost RM, Dao L, et al. Binocular iPad Game vs Patching for Treatment of Amblyopia in Children: A Randomized Clinical Trial. JAMA Ophthalmol 2016;134:1402-8
38 Li SL, Reynaud A, Hess RF, et al. Dichoptic movie viewing treats childhood amblyopia. J Aapos 2015;19:401-5.

39 Žiak P, Holm A, Halička J, et al. Amblyopia treatment of adults with dichoptic training using the virtual reality oculus rift head mounted display: preliminary results. $B M C$ Ophthalmol 2017;17:105.

40 Vedamurthy I, Nahum M, Bavelier D, et al. Mechanisms of recovery of visual function in adult amblyopia through a tailored action video game. Sci Rep 2015;5:8482.

41 Vedamurthy I, Nahum M, Huang SJ, et al. A dichoptic custom-made action video game as a treatment for adult amblyopia. Vision Res 2015;114:173-87.

42 Eastgate RM, Griffiths GD, Waddingham PE, et al. Modified virtual reality technology for treatment of amblyopia. Eye 2006;20:370-4.

43 Waddingham PE, Butler TK, Cobb SV, et al. Preliminary results from the use of the novel Interactive binocular treatment (I-BiT) system, in the treatment of strabismic and anisometropic amblyopia. Eye 2006;20:375-8.

44 Cleary M, Moody AD, Buchanan A, et al. Assessment of a computer-based treatment for older amblyopes: the Glasgow Pilot Study. Eye 2009;23:124-31.

45 Herbison N, Cobb S, Gregson R, et al. I-BiT study group. Interactive binocular treatment (I-BiT) for amblyopia: results of a pilot study of 3D shutter glasses system. Eye 2013:27:1077-83.

46 Herbison N, MacKeith D, Vivian A, et al. Randomised controlled trial of video clips and interactive games to improve vision in children with amblyopia using the I-BiT system. Br J Ophthalmol 2016;100:1511-6.

47 Scheiman MM, Hertle RW, Beck RW, et al. Pediatric Eye Disease Investigator Group. Randomized trial of treatment of amblyopia in children aged 7 to 17 years. Arch Ophthalmol 2005;123:437-47

48 luvone PM, Tigges M, Fernandes A, et al. Dopamine synthesis and metabolism in rhesus monkey retina: development, aging, and the effects of monocular visual deprivation. Vis Neurosci 1989:2:465-71.

49 Gottlob I, Stangler-Zuschrott E. Effect of levodopa on contrast sensitivity and scotomas in human amblyopia. Invest Ophthalmol Vis Sci 1990;31:776-80.

50 Repka MX, Kraker RT, Dean TW, et al. Pediatric Eye Disease Investigator Group. A randomized trial of levodopa as treatment for residual amblyopia in older children. Ophthalmology 2015:122:874-81.

51 Sofi IA, Gupta SK, Bharti A, et al. Efficiency of the occlusion therapy with and without levodopa-carbidopa in amblyopic children-A tertiary care centre experience. Int J Health Sci 2016;10:249-57.

52 Pescosolido N, Stefanucci A, Buomprisco G, et al. Amblyopia treatment strategies and new drug therapies. J Pediatr Ophthalmol Strabismus 2014;51:78-86.

53 Secades JJ. CDP-choline update and review of its pharmacology and clinical use. Methods Find Exp Clin Pharmacol 2001;23:1-53.

54 Pecori Giraldi J, Virno M, Covelli G, et al. Therapeutic value of citicoline in the treatment of glaucoma (computerized and automated perimetric investigation). Int Ophthalmol 1989;13(1-2):109-12.

55 Pawar PV, Mumbare SS, Patil MS, et al. Effectiveness of the addition of citicoline to patching in the treatment of amblyopia around visual maturity: a randomized controlled trial. Indian J Ophthalmol 2014;62:124-9.

56 Campos EC1, Bolzani R, Schiavi C, Baldi A, Porciatti V. Cytidin-5'-diphosphocholine enhances the effect of part-time occlusion in amblyopia. Doc Ophthalmol 19961997;93:247-63

57 Campos EC, Schiavi C, Benedetti P, et al. Effect of citicoline on visual acuity in amblyopia: preliminary results. Graefes Arch Clin Exp Ophthalmol 1995;233:307-12.

58 Fresina M, Dickmann A, Salerni A, et al. Effect of oral CDP-choline on visual function in young amblyopic patients. Graefes Arch Clin Exp Ophthalmol 2008;246:143-50.

59 Nahata MC, Morosco RS, Leguire LE. Development of two stable oral suspensions of levodopa-carbidopa for children with amblyopia. J Pediatr Ophthalmol Strabismus 2000:37:333-7.

60 Dadeya S, Vats P, Malik KP. Levodopa/carbidopa in the treatment of amblyopia. J Pediatr Ophthalmol Strabismus 2009:46:87-90.

61 Campos EC, Fresina M. Medical treatment of amblyopia: present state and perspectives. Strabismus 2006;14:71-3.

62 Ding J, Levi DM. Recovery of stereopsis through perceptual learning in human adults with abnormal binocular vision. Proc Natl Acad Sci U S A 2011;108:E733-41. 